\title{
Endogenously Synthesized Nitric Oxide Prevents Endotoxin-induced Glomerular Thrombosis
}

\author{
Pamela J. Shultz and Leopoldo Raij \\ Department of Medicine, Veterans Administration Medical Center, and University of Minnesota, Minneapolis, Minnesota 55417
}

\begin{abstract}
Escherichia coli endotoxin (LPS) can induce the clinical syndrome of septic shock and renal cortical necrosis and can stimulate nitric oxide (NO) production from macrophages, vascular smooth muscle, and glomerular mesangial cells in vitro. NO is an endogenous vasodilator, which also inhibits platelet aggregation and adhesion. We therefore sought to determine whether LPS would stimulate NO production in vivo and, if so, whether this NO would modulate endotoxin-induced glomerular thrombosis. The stable $\mathrm{NO}$ endproducts, $\mathrm{NO}_{2}$ and $\mathrm{NO}_{3}$, were measured in serum and urine collections from rats during baseline and after injection of LPS, with or without substances that modulate $\mathrm{NO}$ synthesis. The urinary excretion of $\mathrm{NO}_{2} / \mathrm{NO}_{3}$ was $1,964 \pm 311 \mathrm{~nm} / 8 \mathrm{~h}$ during the baseline and increased to $6,833 \pm 776 \mathrm{~nm} / 8 \mathrm{~h}$ after a single intraperitoneal injection of 0.1 $\mathrm{mg} / \mathrm{kg}$ LPS $(P<0.05)$. The serum concentration of $\mathrm{NO}_{2} /$ $\mathrm{NO}_{3}$ also significantly increased after LPS injection. Both the urine and serum stimulation was significantly prevented by the NO synthesis inhibitor, $\boldsymbol{N}_{w}$-nitro-L-arginine methyl ester (LNAME). L-Arginine, given with LPS + L-NAME significantly restored the $\mathrm{NO}_{2} / \mathrm{NO}_{3}$ levels in the urine. Ex vivo incubation of tissues from rats treated with LPS demonstrated NO production by the aorta, whole kidney, and glomeruli, but not cortical tubules. Histological examination of kidneys from rats given either LPS or L-NAME alone revealed that 2 and $4.5 \%$ of the glomeruli contained capillary thrombosis, respectively. In contrast, rats given LPS + L-NAME developed thrombosis in 55\% of glomeruli $(P<0.001)$, which was significantly prevented when $L$-arginine was given concomitantly. We conclude that LPS stimulates endogenous production of $\mathrm{NO}$ in vivo and that this NO is critical in preventing LPS-induced renal thrombosis. (J. Clin. Invest. 1992. 90:1718-1725.) Key words: nitrate $\bullet$ nitrite • cyclic 3',5'-guanosine monophosphate • cortical necrosis • fibrin
\end{abstract}

\section{Introduction}

Escherichia coli LPS is an endotoxin that has been shown to cause the clinical syndrome of septic shock (1). In addition, clinical and experimental endotoxemia is often associated with intravascular coagulation, such as renal cortical necrosis $(2,3)$. Endothelial injury, activation of the coagulation cascade, and

Address correspondence to Pamela J. Shultz, M.D., Renal Section (111J), Veterans Administration Medical Center, 1 Veterans Drive, Minneapolis, MN 55417.

Received for publication 31 January 1992 and in revised form 26 May 1992.

The Journal of Clinical Investigation, Inc.

Volume 90, November 1992, 1718-1725 platelet aggregation have all been shown to contribute to the vascular fibrin deposition $(1,3,4)$. Many substances, including cytokines, prostaglandins, vasoactive, and procoagulant factors have been shown to be stimulated by LPS in vitro and in vivo and may contribute to the clinical effects of LPS (1). However, the secondary mediator (or mediators) responsible for the pathological effects of LPS has not been determined. Identification of this mediator could provide a unifying mechanism to explain the diverse effects of LPS and could suggest possible therapeutic interventions of benefit in endotoxemia and septic shock.

A number of recent studies have led to the hypothesis that nitric oxide (NO) could be a secondary mediator of endotoxemia. NO is an endogenously produced, locally acting vasodilator whose cellular effects appear to be signaled via stimulation of cyclic 3',5'-guanosine monophosphate (cGMP) $(5,6)$. NO has also been shown to inhibit platelet aggregation by itself, as well as synergistically with prostacyclin, another substance of endothelial origin (7). In addition, NO inhibits platelet adhesion to damaged endothelium (8). LPS, as well as tumor necrosis factor (TNF) ${ }^{1}$ and other cytokines, can stimulate production of NO by macrophages and vascular smooth muscle cells in vitro via an inducible $\mathrm{Ca}^{++}$-independent NO synthase (9, 10 ). We have demonstrated that rat glomerular mesangial cells in culture also make NO when incubated with LPS and cytokines and that the endogenously produced NO results in autocrine stimulation of cGMP in these cells (11). Furthermore, tissue homogenates from the liver and lung of rats treated with LPS have been shown to have NO synthase activity (12). Recently, Kilbourn et al. (13) found that inhibition of NO synthesis with the L-arginine analogue, NG-methyl-L-arginine would inhibit TNF-induced hypotension in dogs. Since much evidence suggests that the systemic hemodynamic consequences of endotoxemia are in great part mediated by TNF (14), the latter study provides strong evidence that NO could be a secondary mediator and perhaps a final pathway for LPS-induced vasodilatation and shock. Finally, a brief report by Petros et al. (15) described a beneficial hemodynamic effect of NO synthesis inhibition in two patients with life-threatening septic shock.

However, in addition to the potential deleterious systemic effects of NO production in endotoxemia, a critical amount of locally produced NO may be necessary for the maintenance of organ perfusion and the prevention of vascular thrombosis. In the present studies, the effects of a single intraperitoneal injection of LPS was examined in the rat, a species that is generally resistant to developing glomerular injury after a single exposure to LPS. We have determined the effects of this treatment on the in vivo synthesis of NO, by measuring the serum and

1. Abbreviations used in this paper: D-ARG, D-arginine; L-ARG, L-arginine; L-NAME, $N_{\mathrm{w}}$-nitro-L-arginine methyl ester; NS, normal saline; PAS, periodic acid Schiff; TNF, tumor necrosis factor. 
urine levels of the stable $\mathrm{NO}$ metabolites, nitrite $\left(\mathrm{NO}_{2}\right)$ and nitrate $\left(\mathrm{NO}_{3}\right)$, and by measuring urinary cGMP levels. We have also assessed the effects of the NO synthesis inhibitor, $N_{\mathrm{w}}$-nitro-L-arginine methyl ester (L-NAME), and the NO precursor, L-arginine (L-ARG), on the LPS-induced changes in these biochemical measurements. In addition, we have examined the histological changes within the kidney after exposure to these substances. We have found that a single injection of LPS resulted in endogenous production of NO. Moreover, although neither LPS or L-NAME alone caused glomerular injury, inhibition of LPS-stimulated NO synthesis resulted in loss of renal function and histological evidence of fibrin deposition and glomerular thrombosis.

\section{Methods}

Male Sprague-Dawley rats (Harlan Sprague Dawley Inc., Indianapolis, $\mathrm{IN}$ ), weighing between 250 and $300 \mathrm{~g}$ were used for all experiments.

\section{In vivo experiments}

Serum and urine samples from the rats were obtained on two consecutive days in the following manner: Animals were placed in metabolic cages and 8-h urine collections were obtained for measurement of $\mathrm{NO}_{2} / \mathrm{NO}_{3}$, creatinine, and protein. Animals were allowed free access to water but were not given food during the urine collections. At the end of this period, serum was obtained from a tail incision for $\mathrm{NO}_{2} /$ $\mathrm{NO}_{3}$ and creatinine. On the first day, no treatment was given and these samples are labeled as baseline. On the next day, animals were given intraperitoneal injections following the protocols outlined below and experimental serum and urine samples were obtained in the same manner as the baseline samples. Four different groups of animals were studied:

Group I, LPS. Rats $(n=15)$ in this group received a single intraperitoneal injection of LPS (serotype 0127:B8, Difco Laboratories Inc., Detroit MI) at a dose of $0.1 \mathrm{mg} / \mathrm{kg}$ given at time 0 .

Group II, LPS + L-NAME. These rats $(n=12)$ also received a single intraperitoneal dose of LPS $(0.1 \mathrm{mg} / \mathrm{kg})$ at time 0 , but in addition were given the competitive inhibitor of NO synthesis, L-NAME (Sigma Chemical Co., St. Louis, MO) $75 \mathrm{mg} / \mathrm{kg}$ i.p., $30 \mathrm{~min}$ before, simultaneously with, and 2, 4, and $6 \mathrm{~h}$ after the LPS.

Group III, $L P S+L-N A M E+L-A R G$. These rats $(n=8)$ were given LPS and L-NAME as above and in addition were given $300 \mathrm{mg} / \mathrm{kg}$ i.p. L-ARG (Sigma Chemical Co.) concomitantly with each injection of L-NAME. L-ARG ( $1 \% \mathrm{wt} / \mathrm{vol})$ was also given in the drinking water of these animals, beginning $12 \mathrm{~h}$ before the experimental period.

Group IV, $L-N A M E$. These rats $(n=5)$ were given L-NAME in the same concentration and at the same times as described above, however, $1 \mathrm{~cm}^{3} / \mathrm{kg}$ i.p. of sterile normal saline (NS) was given instead of LPS at time 0 .

All substances were dissolved in saline and prepared fresh each day. In preliminary experiments, it was determined that intraperitoneal injections of saline in the same volume and times as described above had no effect on the parameters measured in our study.

At the end of the 8-h experimental period, the rats were anesthesized and killed by exsanguination, and the kidney tissue was prepared for light and immunofluorescence microscopy as previously described (16). Formalin-fixed, paraffin-embedded 4- $\mu \mathrm{m}$ sections were stained with periodic acid Schiff (PAS) whereas frozen sections were treated with FITC-labeled goat anti-rat polyclonal antibody against fibrinogen (Cappel Laboratories, Durham, NC). The PAS sections were examined in a blinded manner and the percent of glomeruli showing any PAS-positive material in the glomerular capillaries was determined. At least 50 glomeruli were examined from each kidney. Immunofluorescence microscopy was performed on selected tissues to confirm the nature of the PAS-positive material observed on light microscopy. In addition to the groups outline above, the renal histology was also exam- ined in five rats given LPS plus L-NAME plus D-arginine (D-ARG), the biologically inactive enantiomer of L-ARG. D-ARG was given in the same concentration and at the same times as the L-ARG described in group III.

Additional rats underwent placement of an indwelling femoral artery catheter just before the experimental period in order to obtain intraarterial pressure readings. Animals were anesthetized with 5-secbutyl-5-ethyl-2-thiobarbituric acid (Inactin, $100 \mathrm{mg} / \mathrm{kg}$ i.p.) and a PE50 catheter was placed in the femoral artery and then tunneled subcutaneously to an exit site at the dorsum of the neck, using sterile techniques. The catheter was filled with heparinized saline to maintain patency and flushed with $0.1 \mathrm{~cm}^{3}$ after each blood pressure reading. After recovery from the anesthesia, animals were injected with LPS alone, LPS + L-NAME, or L-NAME alone ( $n=4$ animals in each group) as described above for groups I, II, and IV, respectively. The arterial catheter was intermittently connected to a pressure transducer (TNF transducer; Gould Inc., Oxnard, CA) and mean intraarterial blood pressures were obtained before the first injection, $30 \mathrm{~min}$ later, and every $2 \mathrm{~h}$ thereafter for the next $8 \mathrm{~h}$.

\section{Ex vivo experiments}

For these studies, additional rats were treated with either normal saline ( $1 \mathrm{~cm}^{3} / \mathrm{kg}$ i.p.), LPS (as for group I of the in vivo experiments), or LPS + L-NAME (as for group II of the in vivo experiments). Urine was collected for $8 \mathrm{~h}$ and the amount of cGMP excreted by each animal was measured. At the end of the 8-h protocol, tissues from these animals were removed for ex vivo incubations. Rats were anesthetized with Inactin ( $100 \mathrm{mg} / \mathrm{kg}$ i.p.), and, using sterile technique, a midline incision was performed to expose the aorta at the level of the renal arteries. A 22-gauge needle was placed in the aorta with the tip positioned between the left and right renal artery. The aorta was clamped above the level of the renal arteries and both renal veins were cut to facilitate drainage. The kidneys were perfused gently with sterile PBS until they blanched and were then removed. One kidney from each rat was sliced in 2-mm sections. Glomeruli were isolated from the other kidney using a series of nylon sieves as previously described (17). The cortical tissue suspension remaining after harvesting the glomeruli, containing mostly fragments of tubules, was incubated separately. The thoracic aorta from each rat was also removed, washed with sterile PBS, cleaned of fascia, and cut into 4-mm segments, using care not to damage the endothelium. The aortae, whole kidney slices, isolated glomeruli, and cortical tubules were pooled from all the rats in each group and placed in Eagle's MEM without phenol red, supplemented with L-ARG (final concentration $=1 \mathrm{mM}$ ) and $10 \%$ fetal calf serum without any further addition of LPS or L-NAME. The endotoxin contamination in this media was $<0.01 \mathrm{pg} / \mathrm{ml}$ by Limulus assay (11). Tissues were incubated at $37^{\circ} \mathrm{C}$ in $95 \% \mathrm{O}_{2} / 5 \% \mathrm{CO}_{2}$ for $24 \mathrm{~h}$. At the end of this time, the medium was removed from the tissues and frozen at $-20^{\circ} \mathrm{C}$ until it was assayed in duplicate for $\mathrm{NO}_{2} / \mathrm{NO}_{3}$. Background $\mathrm{NO}_{2} / \mathrm{NO}_{3}$ levels in medium not exposed to tissues was subtracted from the sample results which were then factored for the wet weight of the tissue in each dish.

\section{Creatinine, urinary protein excretion, and cGMP assays}

Serum and urine creatinine obtained from the in vivo experiments were measured using a Creatinine Analyser 2 (Beckman Instruments Inc., Fullerton, $\mathrm{CA}$ ) and urine protein was measured by the Coomassie dye method (Bio-Rad Laboratories, Richmond, CA). cGMP was assayed on 8-h urine collections from the three groups of animals used for the ex vivo experiments using a radioimmunoassay, as we have previously described $(11,17)$. Urinary excretion of these substances was calculated by multiplying the measured concentration in the urine by the total urine volume during the 8-h collection. Creatinine clearance was calculated as the urinary excretion of creatinine divided by the plasma creatinine.

\section{$\mathrm{NO}_{2} / \mathrm{NO}_{3}$ assay}

Serum and urine samples, as well as the media from the ex vivo tissue incubations were assayed for the stable end products of $\mathrm{NO}, \mathrm{NO}_{2}$, and 
$\mathrm{NO}_{3}$. Samples were first incubated with $E$. coli nitrate reductase to convert the $\mathrm{NO}_{3}$ in the samples to $\mathrm{NO}_{2}$, as described by Bartholomew (18) and Granger et al. (19). To prepare this enzyme, E. coli (ATCC 25922 ) were grown for $18 \mathrm{~h}$ under anaerobic conditions, washed, resuspended in PBS, and frozen at $-70 \mathrm{C}$. until use. The samples were incubated with enzyme in a ratio of 50:1 (sample/enzyme) in Hepes-ammonium formate buffer for $1 \mathrm{~h}$ at $37^{\circ} \mathrm{C}$. In preliminary assays, this ratio and time were found to give maximum and virtually complete reduction of $\mathrm{NO}_{3}$ without any significant effect on $\mathrm{NO}_{2}$ levels of standards as well as samples. After the enzyme incubation, the total $\mathrm{NO}_{2}$ in the samples (representing both $\mathrm{NO}_{2}$ and the reduced $\mathrm{NO}_{3}$ ) was measured using the Griess reagent, as previously described by ourselves (11) and Green et al. (20). Known concentrations of $\mathrm{NaNO}_{2}$ and $\mathrm{NaNO}_{3}$ were used as standards in each assay.

\section{Statistics}

Results were analyzed by comparing the experimental data from each group using analysis of variance.

\section{Results}

Rats were assigned to the groups at random and the baseline values for all the parameters measured were not different between any of the groups. For this reason, the baseline values for body weight, urine volume, creatinine clearance, and urinary protein excretion were combined for analysis (Table I). Animals treated with a single intraperitoneal injection of LPS showed no obvious illness, even when observed for longer than the 8-h study period. In contrast, rats treated with LPS + LNAME appeared ill and lethargic and $25 \%$ of the animals receiving this protocol expired before the end of the 8-h experimental period. Samples from animals that expired were not included in the analysis and additional rats were given this protocol to replace those that expired. There were no deaths among animals receiving any of the other experimental protocols, including those receiving L-NAME alone.

Intraarterial blood pressure. The average of the mean intraarterial blood pressures obtained before treatment was not significantly different in the animals that received LPS alone $(129 \pm 4)$, LPS + L-NAME $(133 \pm 3)$, or L-NAME alone (121 \pm 2$)$ ( mean $\pm \mathrm{SE}, n=4$ animals in each group). A single dose of LPS $(0.1 \mathrm{mg} / \mathrm{kg}$ i.p.) caused no significant change in mean blood pressure when measured $1,3,5$, or $7 \mathrm{~h}$ later (data not shown). In contrast, animals receiving LPS + L-NAME or L-NAME alone were found to have significant increases in mean intraarterial blood pressure within $30 \mathrm{~min}$ ( $163 \pm 5$ and $156 \pm 2 \mathrm{mmHg}$, respectively). The blood pressure was sustained at similar levels in these two groups of rats for the entire experimental period. At the end of $7 \mathrm{~h}$, the mean intraarterial blood pressure was $128 \pm 1$ in the LPS group, $162 \pm 2$ in the LPS + LNAME group, and $162 \pm 4$ in the L-NAME alone group. The latter two values are not significantly different from each other, but both are significantly higher than LPS alone $(P<0.05)$.

Urine volume, creatinine clearance, and protein excretion. The body weight, urine volume, creatinine clearance, and urinary protein excretion values at baseline and after the experimental periods for the four groups of animals are shown in Table I. There was no significant effect of any of the experimental protocols on body weight. Urine volume was significantly increased in all experimental groups compared with baseline, which may be partially related to the NS in the injections. However, animals that received L-NAME, either with LPS, with LPS + L-ARG, or alone, had significantly greater diuresis than animals that received only LPS without L-NAME. This effect is likely due to the increase in blood pressure induced by LNAME, as described above, leading to a pressure natriuresis. The mean creatinine clearance during the baseline period (all groups) was $1.09 \pm .09 \mathrm{ml} / \mathrm{min}$ and did not change significantly during the experimental period in the group which received LPS ( Table I). In contrast, LPS + L-NAME rats had a significant reduction in creatinine clearance compared with both baseline and rats that received LPS alone $(P<0.01$ vs. baseline, $P<0.05$ vs. LPS alone). When L-ARG was given in addition to LPS + L-NAME, partial restoration of the creatinine clearance towards baseline was observed. L-NAME alone tended to reduce creatinine clearance, similar to what has been reported with the acute intrarenal administration of NO synthesis inhibitors (21), but this reduction was not as great as that observed with LPS + L-NAME and did not reach statistical significance.

Urinary protein excretion (shown in Table I) during the baseline period was negligible and was not affected by a single injection of LPS alone. L-NAME, however, caused a marked increase in protein excretion, either when given with LPS $(88 \pm 15 \mathrm{mg} / 8 \mathrm{~h})$ or when given alone $(63 \pm 27 \mathrm{mg} / 8 \mathrm{~h})$. The animals treated with LPS + L-NAME + L-ARG had intermediate levels of protein excretion $(29 \pm 5 \mathrm{mg} / 8 \mathrm{~h}, n=8)$.

Urinary excretion and serum concentrations of $\mathrm{NO}_{2} / \mathrm{NO}_{3}$. Fig. 1 compares the urinary excretion of $\mathrm{NO}_{2} / \mathrm{NO}_{3}$ at baseline and after the four different experimental protocols. The baseline urinary excretion of $\mathrm{NO}_{2} / \mathrm{NO}_{3}$ was not different between any of the four groups, so this data was pooled and is shown as a single baseline value in Fig. 1. The value for the baseline urinary $\mathrm{NO}_{2} / \mathrm{NO}_{3}$ excretion was $1,964.4 \pm 311 \mathrm{nmol} / 8 \mathrm{~h}$. LPS injection significantly increased this value to $6,833.0 \pm 776(P$ $<0.001)$. Treatment of the animals with the NO synthesis inhibitor L-NAME completely inhibited the increase in urinary

Table I. Effect of LPS and NO Synthesis Inhibition on Body Weight and Renal Functional Parameters

\begin{tabular}{lccccc}
\hline \multicolumn{1}{c}{ Group } & $n$ & Body weight & Urine volume & CrCl & UV prot \\
\hline & & $g$ & $m g / m h$ & $m g / 8 h$ \\
Baseline (mean of 4 groups) & 28 & $273.4 \pm 3.7$ & $3.3 \pm 0.4$ & $1.09 \pm 0.09$ & $4 \pm 0.4$ \\
LPS & 7 & $265.7 \pm 8.8$ & $8.3 \pm 1.1^{*}$ & $1.12 \pm 0.09$ & $0.57 \pm 0.05^{* \neq}$ \\
LPS + L-NAME & 8 & $268.1 \pm 5.7$ & $14.9 \pm 1.0^{* \neq}$ & $88 \pm 15^{* \neq}$ \\
LPS + L-NAME + L-ARG & 8 & $295.6 \pm 4.5$ & $16.2 \pm 1.1^{*}$ & $0.72 \pm 0.11$ & $29 \pm 5^{5}$ \\
L-NAME alone & 5 & $273.0 \pm 8.9$ & $12.8 \pm 0.5^{*}$ & $0.70 \pm 0.15$ & $63 \pm 27^{*}$
\end{tabular}

Values are mean \pm SE. See text for definitions of groups. $\mathrm{Cr}$, creatinine; $\mathrm{CrCl}$, creatinine clearance; $\mathrm{UV}$ prot, urinary excretion of protein in $8 \mathrm{~h}$. ${ }^{*} P<0.01$ vs. baseline. ${ }^{\ddagger} P<0.05$ vs. LPS. ${ }^{B} P<0.01$ vs. LPS + L-NAME. 


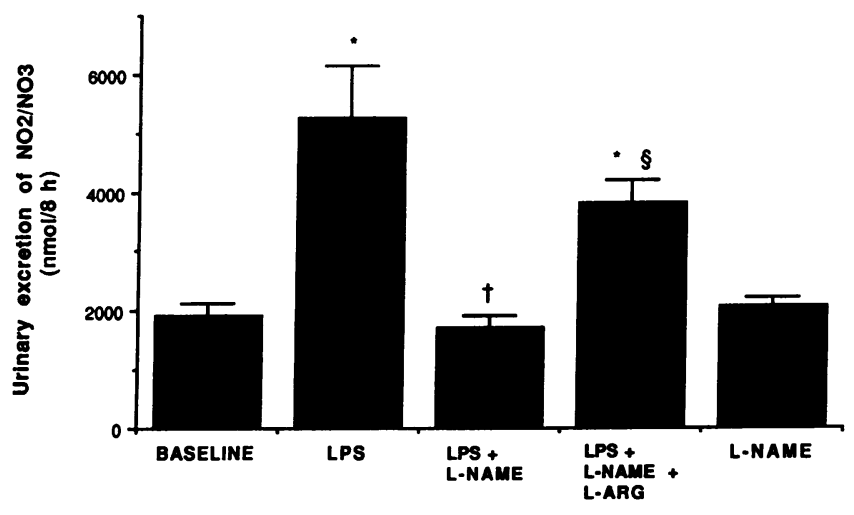

Figure 1. Urinary excretion of $\mathrm{NO}_{2} / \mathrm{NO}_{3}$ during baseline period and in rats receiving LPS $(n=15)$, LPS + L-NAME $(n=16)$, LPS + L-NAME + L-ARG $(n=8)$, or L-NAME alone $(n=5)$. See text for concentrations and timing of injections of the various substances. Total $\mathrm{NO}_{2}$ (after reduction of $\mathrm{NO}_{3}$ ) was measured using the Griess reagent. Mean $\pm \mathrm{SE} ;{ }^{*} P<0.001$ versus baseline; ${ }^{\dagger} P<0.001$ versus LPS; ${ }^{8} P<0.001$ versus LPS + L-NAME.

excretion of $\mathrm{NO}_{2} / \mathrm{NO}_{3}$ induced by LPS $(P<0.01 \mathrm{LPS}+\mathrm{L}-$ NAME vs. LPS alone; Fig. 1). When animals were treated with L-ARG in addition to L-NAME and LPS, significant reversal of this inhibition could be seen $(P<0.001)$. L-NAME given alone, without LPS, had no effect on urine $\mathrm{NO}_{2} / \mathrm{NO}_{3}$. The mean value for urinary excretion of $\mathrm{NO}_{2} / \mathrm{NO}_{3}$ in this latter group was $2,054 . \pm 162$, which was not significantly different than the values obtained during the baseline period.

The concentration of $\mathrm{NO}_{2} / \mathrm{NO}_{3}$ in the serum of rats at baseline and after completion of each of the experimental protocols is shown in Fig. 2. The baseline serum $\mathrm{NO}_{2} / \mathrm{NO}_{3}$ level, pooled from all four groups, was $131.1 \pm 9 \mu \mathrm{M} .8 \mathrm{~h}$ after a single, intraperitoneal injection of LPS, the $\mathrm{NO}_{2} / \mathrm{NO}_{3}$ concentration rose significantly to $546.1 \pm 92 \mu \mathrm{M}(P<0.001)$. This increase was significantly prevented in rats given LPS + L-NAME $(P$ $<0.05$ ). In contrast to the urinary $\mathrm{NO}_{2} / \mathrm{NO}_{3}$ excretion, the mean serum $\mathrm{NO}_{2} / \mathrm{NO}_{3}$ levels in rats receiving $\mathrm{LPS}+\mathrm{L}-\mathrm{NAME}$

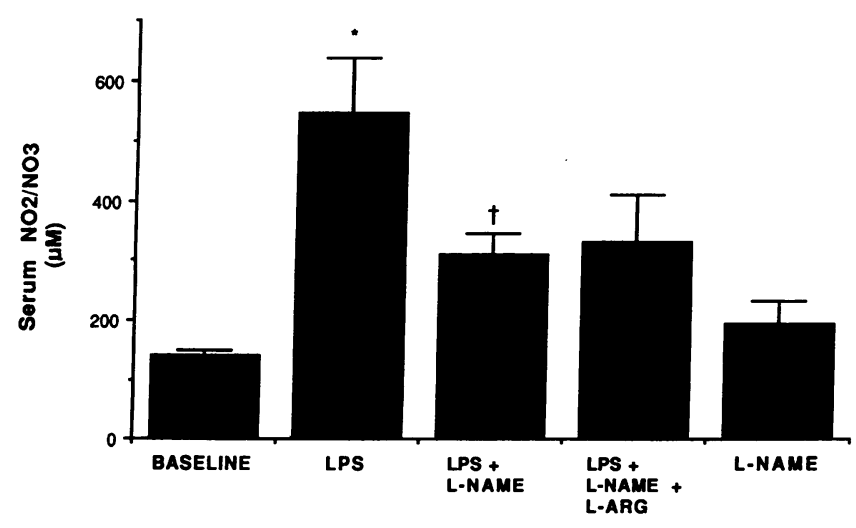

Figure 2. Concentrations of $\mathrm{NO}_{2} / \mathrm{NO}_{3}$ in serum from rats at baseline and $8 \mathrm{~h}$ after receiving LPS $(n=15)$, LPS + L-NAME $(n=12)$, LPS + L-NAME + L-ARG $(n=4)$, or L-NAME alone $(n=5)$. See text for concentrations and timing of injections of the various substances. Total $\mathrm{NO}_{2}$ (after reduction of $\mathrm{NO}_{3}$ ) was measured using the Griess reagent. Mean $\pm \mathrm{SE} ;{ }^{*} P<0.001$ versus baseline; ${ }^{\dagger} P<0.05$ versus LPS.

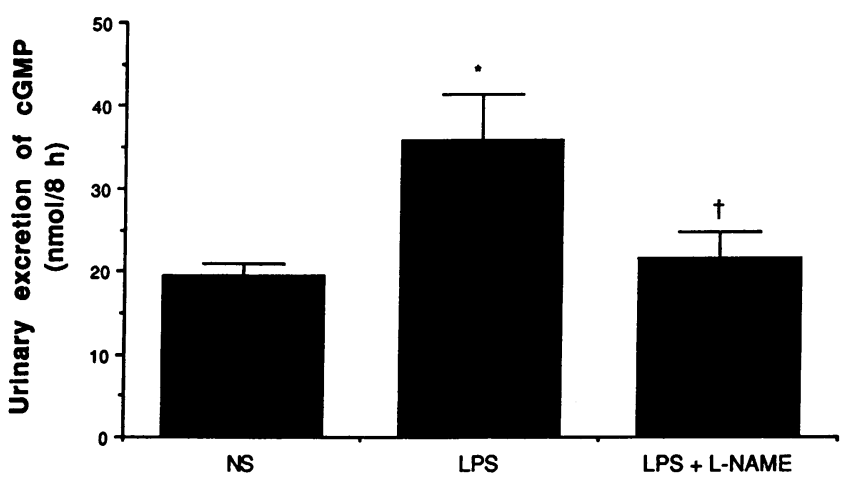

Figure 3. Urinary excretion of CGMP during the experimental period in rats given NS in a volume equal to that given in the other groups, LPS or LPS + L-NAME. See text for concentrations and timing of injections. Values are mean \pm SE; $n=6$ rats in each group. ${ }^{*} P<0.05$ versus NS; ${ }^{\dagger} P<0.05$ versus LPS.

+ L-ARG was not different than the LPS + L-NAME group. Lastly, L-NAME alone did not significantly alter serum $\mathrm{NO}_{2} /$ $\mathrm{NO}_{3}$ levels compared with baseline.

Urinary cGMP excretion. We also measured urinary cGMP excretion on 8-h urine collections obtained from rats treated with normal saline, LPS, or LPS + L-NAME. Rats injected with saline excreted $19.5 \pm 1.4 \mathrm{nmol}$ cGMP/8 h (Fig. 3) whereas rats treated with a single injection of LPS excreted $35.8 \pm 5.5 \mathrm{nmol}$ cGMP $/ 8 \mathrm{~h}(P<0.01$ vs. NS $)$. Lastly, the rats that received L-NAME + LPS excreted $21.6 \pm 3 \mathrm{nmol}$ cGMP $/ 8$ $h$, a value that is significantly lower than rats receiving LPS alone $(P<0.01$ vs. LPS alone $)$ and no different than rats given saline.

Renal histology. The light microscopic and immunofluorescent findings of a representative glomerulus from a rat treated with a single intraperitoneal injection of $0.1 \mathrm{mg} / \mathrm{kg}$ LPS are shown in Fig. $4 A$ and $B$. Minimal glomerular or tubular abnormalities were seen in these animals and, using polyclonal antibody for rat fibrinogen, fibrin deposition in these glomeruli was quite rare. In contrast, a glomerulus from a rat that received LPS + L-NAME is shown in Fig. 4, $C$ and $D$. Many of the glomeruli from these rats showed deposition of a PAS-positive homogeneous material within the capillary loops, as well as proteinaceous material within Bowman's space and the tubular lumen. In addition, there was tubular epithelial cell swelling and desquamation noted. No significant cellular infiltration or proliferation was noted within the glomerulus or the interstitium. Immunofluorescence microscopy, (Fig. $4 D$ ) confirmed that the material deposited within the glomerular capillary loops was antigenically related to fibrinogen, consistent with fibrin. Most of the glomeruli showing thrombi had diffuse fibrin deposition throughout all the capillary loops, although in some glomeruli the fibrin deposition was segmental.

Fig. 5 depicts the percent of glomeruli containing thrombosis by light microscopy in rats subjected to the four experimental protocols used for the in vivo experiments. Of all the glomeruli examined from rats treated with LPS alone, only $2.2 \pm 2 \%$ (mean $\pm \mathrm{SE}$ ) showed thrombosis whereas rats treated with LPS + L-NAME had $54.6 \pm 11 \%$ of glomeruli thrombosed, most of which was global $(P<0.001$ compared with LPS alone). Rats treated with LPS + L-NAME + L-ARG had significantly less glomerular thrombosis $(16.5 \pm 5 \%)$ than rats treated 

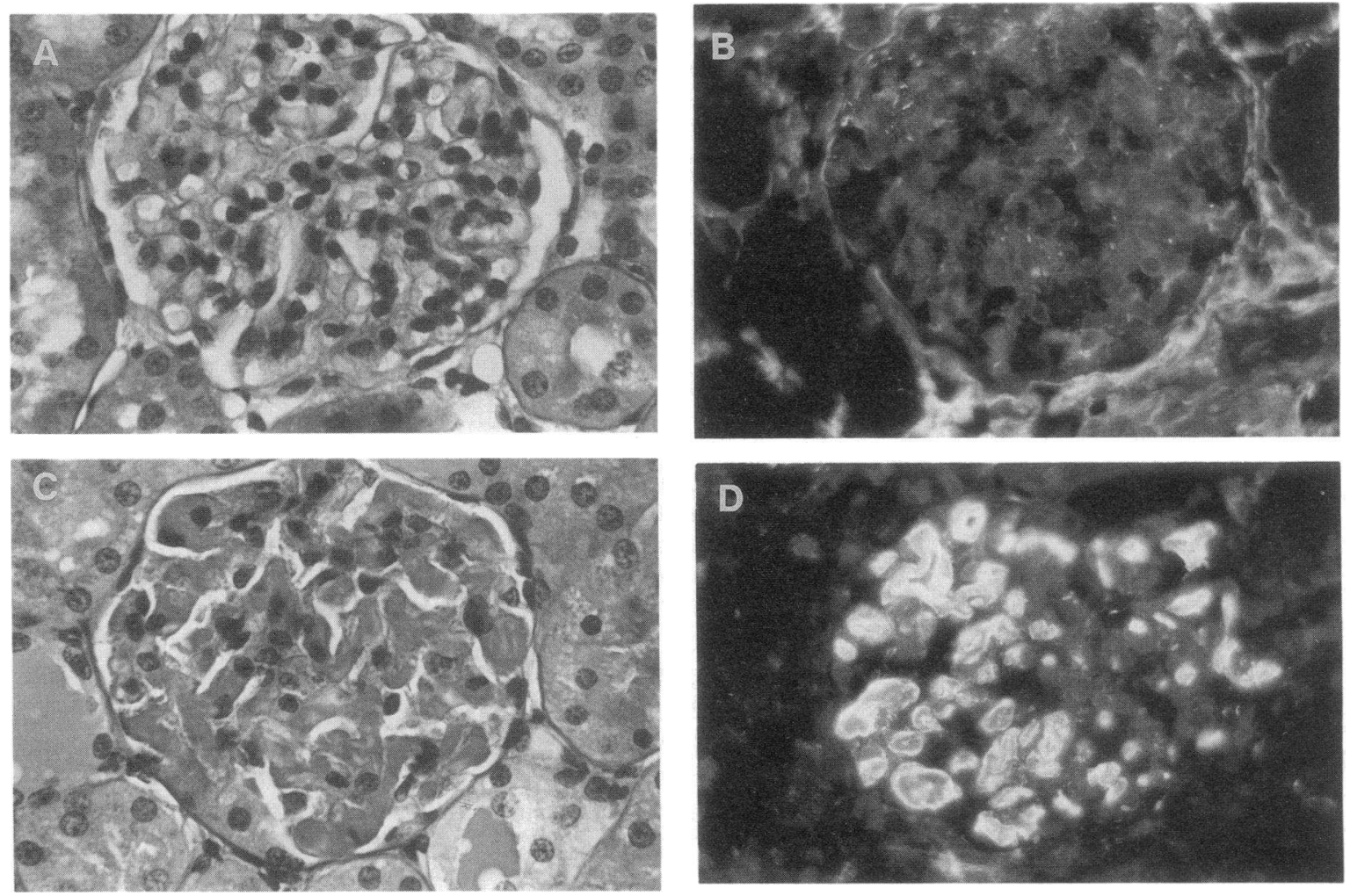

Figure 4. Representative photomicrographs of glomeruli from rats given LPS alone ( $A$ and $B$ ) or LPS + L-NAME $(C$ and $D)$. Kidneys were removed at the end of the 8-h experimental period, and portions were examined under light microscopy with PAS staining $(A$ and $C)$ or processed for immunofluorescence with FITC-labeled antibody to fibrinogen $(B$ and $D$ ). Glomeruli from animals given LPS alone showed no significant changes from normal and negative fluorescence whereas those given LPS + L-NAME showed extensive thrombi within the glomerular capillary loops and markedly positive fluorescent staining (original magnification $\times 400$ ).

with LPS + L-NAME $(P<0.01)$. To rule out the possibility of a nonspecific protective effect of amino acid injection on the observed glomerular injury, five rats were treated with LPS + L-NAME + D-ARG (the biologically inactive enantiomer of L-ARG, which is not a substrate for NO synthesis). These rats were found to have $45.8 \pm 11 \%$ of their glomeruli thrombosed ( $n=5$, data not shown), which was not significantly different than the LPS + L-NAME group. Finally, rats treated with L-

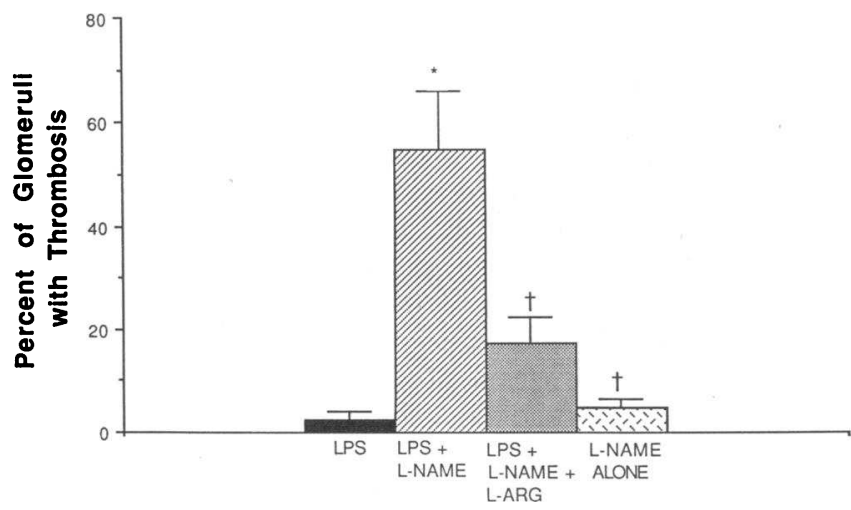

Figure 5. Percent of glomeruli with thrombosis in kidneys from the four experimental groups. 50 glomeruli from each kidney were examined under light microscopy and the results shown are the mean $\pm \mathrm{SE}$ in kidneys from five to eight rats from each group. ${ }^{*} P$ $<0.001$ versus LPS; ${ }^{\dagger} P<0.01$ versus LPS + L-NAME.
NAME alone had $4.6 \pm 2 \%$ of glomeruli thrombosed, which was not significantly different from that observed in rats treated with LPS alone (Fig. 5).

Ex vivo incubations. To determine possible sources of the increased serum and urine $\mathrm{NO}_{2} / \mathrm{NO}_{3}$ seen in rats after LPS treatment, selected tissues were removed at the end of the 8-h experimental period for ex vivo incubations. $\mathrm{NO}_{2} / \mathrm{NO}_{3}$ production during these ex vivo incubations in tissues from rats treated with normal saline (NS), LPS, or LPS + L-NAME is shown in Fig. 6. We find that the aortic segments, kidney slices, and glomeruli from animals treated with LPS all produced more $\mathrm{NO}_{2} / \mathrm{NO}_{3}$ during the 24-h ex vivo incubation than similar tissues taken from animals treated with NS. Aortic segments, whole kidney slices, and glomeruli from LPS-treated rats produced 2.4-, 2.0-, and 48.0-fold more $\mathrm{NO}_{2} / \mathrm{NO}_{3}$, respectively, than the same tissues from NS-treated rats. This induced production of $\mathrm{NO}_{2} / \mathrm{NO}_{3}$ was markedly reduced in the same tissues obtained from rats treated with LPS + L-NAME, confirming that the $\mathrm{NO}_{2} / \mathrm{NO}_{3}$ measured in the media derives from NO. In contrast, the cortical tubules from NS-, LPS-, and LPS + L-NAME-treated rats all produced similar amounts of $\mathrm{NO}_{2} /$ $\mathrm{NO}_{3}$ during the ex vivo incubation (Fig. 6), suggesting that LPS does not stimulate NO production in this tissue.

\section{Discussion}

Our studies demonstrate that a single intraperitoneal injection of LPS increases the levels of the NO end products, $\mathrm{NO}_{2}$ and 


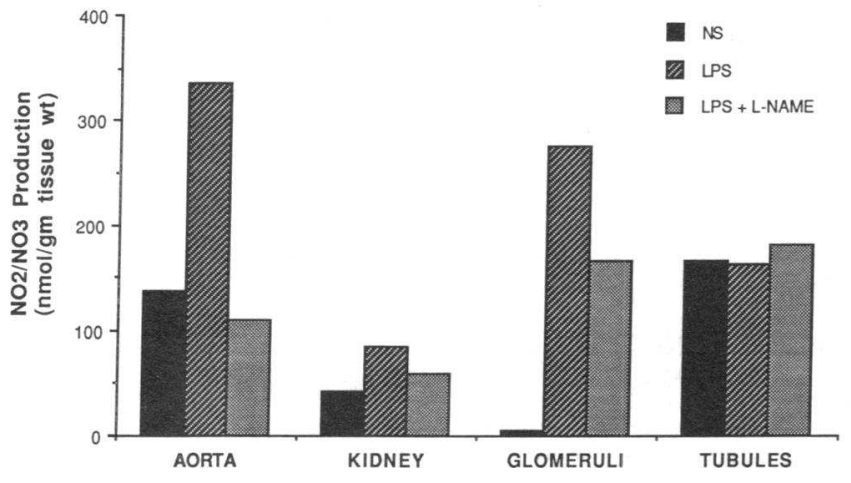

Figure $6 . \mathrm{NO}_{2} / \mathrm{NO}_{3}$ production by rat aortic segments, whole kidney slices, isolated glomeruli, and cortical tubular fragments after ex vivo incubation. Rats were injected in vivo with either NS (solid bars), LPS (hatched bars), or LPS + L-NAME (stippled bars). See text for details of the concentrations and timing of the injections. $8 \mathrm{~h}$ after the injections, rats were killed and tissues were removed and incubated for $24 \mathrm{~h}$ in Eagle's MEM containing $1 \mathrm{mM} \mathrm{L}-\mathrm{ARG}$ and $10 \%$ serum. Total $\mathrm{NO}_{2}$ (after reduction of $\mathrm{NO}_{3}$ ) was measured in the medium and factored for wet weight of the tissue in the same dish. Tissues were pooled from eight rats in one experiment and four rats in a second experiment. Values shown are the means of the results from these two experiments.

$\mathrm{NO}_{3}$, in the serum and urine of rats. The changes in urinary $\mathrm{NO}_{2} / \mathrm{NO}_{3}$ were completely inhibited by the $\mathrm{NO}$ synthesis inhibitor, L-NAME, and significantly restored when rats were given excess L-ARG in addition to LPS + L-NAME. This confirms that the changes in urinary $\mathrm{NO}_{2} / \mathrm{NO}_{3}$ that we detected were due to changes in $\mathrm{NO}$ production. The serum $\mathrm{NO}_{2} / \mathrm{NO}_{3}$ levels were also significantly, although not completely inhibited in rats given LPS + L-NAME. L-ARG failed to reverse this inhibitory effect, but this could be due to the fact that the serum $\mathrm{NO}_{2} / \mathrm{NO}_{3}$ levels represent a single point in time whereas the urine $\mathrm{NO}_{2} / \mathrm{NO}_{3}$, measured on a timed collection, represents a more integrated value, reflecting the accumulated $\mathrm{NO}_{2} / \mathrm{NO}_{3}$ production during the entire 8-h experimental period. Alternatively, it may suggest that exogenous L-ARG is more effective in reversing the inhibitory effect of L-NAME on NO synthesis in the kidney. In either case, our studies demonstrate that LPS given in vivo to rats can induce endogenous production of NO. Our findings are consistent with a previous report in mice (22) and with a recent publication by Hibbs et al. (23), which demonstrated increases in serum and urine $\left[{ }^{15} \mathrm{~N}\right] \mathrm{NO}_{3}$ in humans given $\mathrm{L}-\left[{ }^{15} \mathrm{~N}\right]$ arginine and interleukin-2. This latter study, as well as the present results, establish that urinary $\mathrm{NO}_{2} / \mathrm{NO}_{3}$ excretion, and perhaps to a lesser extent serum concentrations of $\mathrm{NO}_{2} / \mathrm{NO}_{3}$, can be used as markers of $\mathrm{NO}$ production in vivo.

Urinary excretion of cGMP has previously been shown by Tolins et al. (24) to be a marker of NO synthesis after intravenous acetylcholine infusion. In that study, acetylcholine was thought to stimulate NO production via the constitutive NO synthase in the vascular endothelium. In the present study, we show that urinary cGMP excretion also increases after LPS and that this rise is prevented when L-NAME is given with the LPS. These changes in urinary excretion of cGMP are further evidence that LPS induces NO production in vivo. Furthermore, the increase in cGMP excretion suggests that the NO being produced is biologically active since most cellular actions of
NO occur via stimulation of intracellular soluble guanylate cyclase, leading to increases in $\operatorname{cGMP}(6,11,12)$.

Our in vivo studies do not allow us to determine precisely the relative contributions of different tissues to the changes in serum and urine $\mathrm{NO}_{2} / \mathrm{NO}_{3}$ after intraperitoneally injected LPS. However, the ex vivo incubations demonstrate that the aorta, the kidney, and the glomeruli, taken from animals treated in vivo with LPS all produced greater amounts of $\mathrm{NO}_{2} /$ $\mathrm{NO}_{3}$ than tissues removed from animals treated with saline. Of all tissues tested, the glomeruli showed the most dramatic increase in $\mathrm{NO}_{2} / \mathrm{NO}_{3}$ after exposure to LPS. Moreover, the increased production of $\mathrm{NO}_{2} / \mathrm{NO}_{3}$ was inhibited in tissues taken from animals treated with LPS + L-NAME. The stimulatory effect of LPS continued for $24 \mathrm{~h}$ after removing the tissues from the animals, suggesting that either the LPS accumulates in these tissues or, more likely, that an NO synthase enzyme has been induced and continues to synthesize NO even after the stimulus is removed (25).

These results are consistent with the findings of ourselves and others of an inducible NO synthase in vascular smooth muscle and glomerular mesangial cells in culture $(10,11)$. In those studies, $\geq 4 \mathrm{~h}$ of incubation with LPS were required before increases in $\mathrm{NO}_{2} / \mathrm{NO}_{3}$ and cGMP could be detected from the mesangial cells (11). Other investigators have demonstrated an inducible type of NO synthase enzyme, responsive to LPS and other cytokines, in macrophages $(6,26)$. Glomerular endothelial cells also possess an inducible cytokine-stimulated NO synthase, in addition to the well-studied constitutive isoform (27). Thus although several different cell types within the aorta and glomerulus may be contributing to the changes in $\mathrm{NO}_{2} / \mathrm{NO}_{3}$ that we measured, it is clear that the aorta and kidney are major sources of the LPS-induced NO production in vivo in our studies. Whether the constitutive NO synthase enzyme in vascular or glomerular endothelium is also activated under these experimental conditions is difficult to conclude from the present studies.

No significant changes in renal function or histology were detected after the single injection of LPS. This is consistent with many previous studies demonstrating that a single injection of LPS in a normal rat is not sufficient to cause renal cortical necrosis $(2,3,28)$. However, in our rats given the NO synthesis inhibitor L-NAME, in addition to a single injection of LPS, we found a significant reduction in $\mathrm{CrCl}$ as well as thrombosis and fibrin deposition within the glomerular capillaries (Table I and Fig. 4). The histological changes within the kidney are similar to what has been described in the generalized Schwartzman reaction $(3,29)$. Partial restoration of NO production by L-ARG significantly reversed both the decrease in $\mathrm{CrCl}$ and the glomerular thrombosis seen with LPS $+\mathrm{L}-$ NAME. The effect of L-ARG was specific, presumably by overcoming the competitive inhibitory effect of L-NAME, since D-ARG did not reverse the glomerular injury induced by LPS + L-NAME. Importantly, NO synthesis inhibition by itself caused only an insignificant decrease in $\mathrm{CrCl}$ and caused glomerular thromboses in $<5 \%$ of glomeruli. We conclude from these studies that inhibition of the LPS-stimulated NO production leads to glomerular thrombosis and dysfunction whereas inhibition of basal NO production does not. We speculate that direct injury to the vascular endothelium from LPS (4) in addition to loss of NO production are required for glomerular thrombosis to occur. 
Marked increases in proteinuria were also observed in the animals given LPS + L-NAME. However, nearly similar degrees of proteinuria were seen in the rats that received L-NAME alone. Since the latter animals had minimal glomerular damage upon histological examination, it is unlikely that the proteinuria is indicative of renal injury in this case. Rather, it is probable that the proteinuria observed in our model after NO inhibition was due to glomerular hypertension, since both the group treated with L-NAME alone and those treated with LPS + L-NAME had systemic hypertension. In addition, recent micropuncture studies have demonstrated that both acute and chronic inhibition of NO synthesis leads to increased glomerular capillary pressure $(29,30)$ and the latter was also associated with proteinuria in the rat (30).

Most investigators now concur that NO is one of the endothelium-derived relaxing factors and that it plays a role in basal vascular tone, as well as in disease states, such as hypertension and acute renal failure $(24,31,32)$. Most of this work has studied alterations in the constitutive enzyme within the vascular endothelium. However, as discussed above, it is likely that LPS is activating the inducible type NO synthase in the present studies. Although LPS alone did not cause a reduction in systemic blood pressure in our hands, others have suggested that NO produced in response to high circulating levels of endotoxin and/or cytokines may be responsible for vasodilatation and shock sometimes seen in these conditions (12-14). In this way, NO could contribute in a detrimental way to the pathophysiology of endotoxemia. However, local vasodilatation induced by NO could also help to maintain critical organ perfusion during periods of hypoperfusion. In addition, the antiaggregatory and antiplatelet adhesion properties of $\mathrm{NO}(5,7,8)$ could protect against vascular thrombosis in small vessels. Both of these actions of NO, as well as other yet undefined effects, could be protective in endotoxemia and may explain why inhibition of NO production in our model caused extensive glomerular thrombosis and renal dysfunction. A recent study has observed increased thrombosis and necrosis in the livers of rats treated with Corynebacterium parvum + LPS after inhibition of NO production (33). Thus, it is likely that prevention of LPS-induced NO production can lead to capillary thrombosis in a variety of organs and confirms our concept that NO production in response to LPS is a protective or counterregulatory response.

Species and individual variability in the susceptibility to acute and chronic renal failure during endotoxemia is well documented $(2,28)$. Most animal species require a "priming" maneuver before LPS injection to develop glomerular thrombi $(2,3,28)$. In addition, in clinical medicine, it is well known that not all patients with endotoxemia, or even all those with septic shock, develop acute renal failure or cortical necrosis (34). It is possible that an individual's capacity to produce NO in response to LPS, as well as variations in the local response to this mediator, could determine the overall clinical outcome of individuals with endotoxemia.

Recent studies have suggested that inhibition of NO may improve the systemic vasodilitation and hypotension associated with endotoxemia and septic shock $(15,35)$, however, others have suggested that high doses of NO synthesis inhibitors in this setting may increase mortality (36). Our study suggests that inhibition of NO synthesis may induce or potentiate glomerular thrombosis and injury during endotoxemia. There- fore, we conclude that any therapeutic manipulation of NO production in vivo must be undertaken cautiously. A complete understanding of the physiological and pathophysiological as well as the potential therapeutic role of NO in renal disease must await further studies.

\section{Acknowledgments}

The authors thank Dr. Alfred Fish, Department of Pediatrics, University of Minnesota, Minneapolis, MN for performing the immunofluorescence staining and microscopy used in these studies, and Dr. Judit Nagy, Department of Medicine, Veterans Administration Medical Center, Minneapolis, MN for measurement of urinary cGMP. We also acknowledge and appreciate the technical assistance of Ms. Karen Coffee and Ms. Kimberly Lewis.

These studies were supported by funds from the Department of Veterans Affairs Medical Research.

\section{References} 469.

1. Bone, R. C. 1991. The pathogenesis of sepsis. Ann. Intern. Med. 115:457-

2. Beller, F. K. 1969. The role of endotoxin in disseminated intravascular coagulation. Thromb. Diath. Haemorrh. 36:125-149.

3. Raij, L., W. F. Keane, and A. F. Michael. 1977. Unilateral Shwartzman reaction: cortical necrosis in one kidney following in vivo perfusion with endotoxin. Kidney Int. 12:91-95.

4. Gaynor, E., C. A. DeBouvier, and T. H. Spaet. 1970. Vascular lesions: possible pathogenic basis of the generalized Shwartzman reaction. Science (Wash. DC). 170:986-988.

5. Moncada, S., R. M. J. Palmer, and E. A. Higgs. 1991. Nitric oxide: physiology, pathophysiology, and pharmacology. Pharmacol. Rev. 43:109-142.

6. Ignarro, L. J., and P. J. Kadowitz. 1985. The pharmacological and physiological role of cyclic GMP in vascular smooth muscle relaxation. Annu. Rev. Pharmacol. Toxicol. 25:171-191.

7. Radomski, M., R. M. J. Palmer, and S. Moncada. 1987. A comparative pharmacology of endothelium-derived relaxing factor, nitric oxide and prostacyclin in platelets. Br. J. Pharmacol. 92:181-197.

8. Radomski, M. W., R. M. J. Palmer, and S. Moncada. 1987. The role of nitric oxide and CGMP in platelet adhesion to vascular endothelium. Biochem. Biophys. Res. Commun. 148:1482-1489.

9. Marletta, M. A., P. S. Yoon, R. Iyengar, C. D. Leaf, and J. S. Wishnok. 1988. Macrophage oxidation of $L$-arginine to nitrite and nitrate: nitric oxide is an intermediate. Biochemistry. 27:8706-8711.

10. Beasley, D., J. H. Schwartz, and B. M. Brenner. 1991. Interleukin 1 induces prolonged L-arginine dependent cyclic guanosine monophosphate and nitric production in rat vascular smooth muscle cells. J. Clin. Invest. 87:602-608

11. Shultz, P. J., M. A. Tayeh, M. A. Marletta, and L. Raij. 1991. Synthesis and action of nitric oxide in rat glomerular mesangial cells. Am. J. Physiol. 261:F600-F606.

12. Knowles, R. G., M. Salter, S. L. Brooks, and S. Moncada. 1990. Anti-inflammatory glucocorticoids inhibit the induction by endotoxin of nitric oxide synthase in the lung, liver and aorta of the rat. Biochem. Biophys. Res. Commun. 172:1042-1048.

13. Kilbourn, R. G., S. S. Gross, A. Jubran, J. Adams, O. W. Griffith, R. Levi, and R. F. Lodato. 1990. N ${ }^{G}$-Methyl-L-arginine inhibits tumor necrosis factor-induced hypotension: implications for the involvement of nitric oxide. Proc. Natl. Acad. Sci. USA. 87:3629-3632.

14. Michie, H. R., K. R. Manogue, D. R. Spriggs, A. Revhaug, S. O’Dwyer, C. A. Dinarello, A. Cerami, S. M. Wolff, and D. W. Wilmore. 1988. Detection of circulating tumor necrosis factor after endotoxin administration. $N$. Engl. J. Med. 318:1481-1486.

15. Petros, A., D. Bennett, and P. Vallance. 1991. Effect of nitric oxide synthase inhibitors on hypotension in patients with septic shock. Lancet. 338:15571558.

16. Raij, L., S. Azar, and W. Keane. 1984. Mesangial immune injury, hypertension, and progressive glomerular damage in Dahl rats. Kidney Int. 26:137143.

17. Shultz, P. J., A. E. Schorer, and L. Raij. 1990. Effects of endothelium-derived relaxing factor and nitric oxide on rat mesangial cells. Am. J. Physiol. 258:F162-F167.

18. Bartholomew, B. 1984. A rapid method for the assay of nitrate in urine 
using the nitrate reductase enzyme of Escherichia coli. Food Chem. Toxicol 22:541-543.

19. Granger, D. L., J. B. Hibbs, Jr., J. R. Perfect, and D. T. Durack. 1990 Metabolic fate of L-arginine in relation to microbiostatic capability of murine macrophages. J. Clin. Invest. 85:264-273.

20. Green, L. C., D. A. Wagner, J. Glogowski, P. L. Skipper, J. S. Wishnok, and S. R. Tannenbaum. 1982. Analysis of nitrate, nitrite, and ${ }^{15} \mathrm{~N}$ nitrate in biological fluids. Anal. Biochem. 126:131-138

21. Tolins, J. P., and L. Raij. 1991. Effects of amino acid infusion on rena hemodynamics. Role of endothelium-derived relaxing factor. Hypertension (Dallas). 17:1045-1051.

22. Stuehr, D. J., and M. A. Marletta. 1985. Mammalian nitrate biosynthesis: mouse macrophages produce nitrite and nitrate in response to Escherichia coli lipopolysaccharide. Proc. Natl. Acad. Sci. USA. 82:7738-7742.

23. Hibbs, J. B., Jr., C. Westenfelder, R. Taintor, Z. Vavrin, C. Kablitz, R. L. Baranowski, J. H. Ward, R. L. Menlove, M. P. McMurry, J. P. Kushner, et al. 1992. Evidence for cytokine-inducible nitric oxide synthesis from L-arginine in patients receiving interleukin-2 therapy. J. Clin. Invest. 89:867-877.

24. Tolins, J. P., R. M. J. Palmer, S. Moncada, and L. Raij. 1990. Role of endothelium-derived relaxing factor in regulation of renal hemodynamic responses. Am. J. Physiol. 258:H655-H662.

25. Nathan, C. F., and D. J. Stuehr. 1990. Does endothelium-derived nitric oxide have a role in cytokine-induced hypotension? J. Natl. Cancer Inst. (Bethesda). 82:762-772.

26. Hibbs, J. B., Jr., R. R. Taintor, Z. Vavrin, and E. M. Rachlin. 1988. Nitric oxide: a cytotoxic activated macrophage effector molecule. Biochem. Biophys. Res. Commun. 157:87-94.

27. Lamas, S., T. Michel, B. M. Brenner, and P. A. Marsden. 1991. Nitric oxide synthesis in endothelial cells: evidence for a pathway inducible by TNF-a. Am. J. Physiol. 261:C634-C641.

28. Bergstein, J. M., J. R. Hoyer, and A. F. Michael. 1974. Glomerular fibrinolytic activity following endotoxin-induced glomerular fibrin deposition in the pregnant rat. Am. J. Pathol. 75:195-202.

29. Ferrario, R., A. Fogo, K. Takahashi, and K. F. Badr. 1991. Microcirculatory responses to inhibition of nitric oxide (NO) synthesis in normal kidneys and during acute glomerulonephritis in the rat. J. Am. Soc. Nephrol. 2:504. (Abstr.)

30. Baylis, C., L. Samsell, and A. Deng. 1991. A new model of systemic hypertension with high glomerular capillary blood pressure $\left(P_{G C}\right)$ and proteinuria: chronic blockade of endogenous endothelial derived relaxing facto (EDRF). J. Am. Soc. Nephrol. 2:471. (Abstr.)

31. Shultz, P. J., J. P. Tolins, and L. Raij. 1990. Endothelial-derived vasoactive substances and the kidney. Kidney. 23:1-8.

32. Luscher, T. F., and P. M. Vanhoutte. 1988. Hypertension and endothelium-dependent responses. In Vasodilatation-Vascular Smooth Muscle, Peptides, Autononic Nerves, and Endothelium. P. M. Vanhoutte, editor. Raven Press Ltd., New York. 523-529.

33. Billiar, T. R., R. D. Curran, B. G. Harbrecht, D. J. Stuehr, A. J. Demetris and R. L. Simmons. 1990. Modulation of nitrogen oxide synthesis in vivo: $\mathbf{N}^{\mathbf{G}}$ Monomethyl-L-arginine inhibits endotoxin-induced nitrite/nitrate biosynthesis while promoting hepatic damage. J. Leukocyte Biol. 48:565-569.

34. Donohoe, J. F. 1983. Acute bilateral cortical necrosis. In Acute Renal Failure. B. J. Brenner, J. M. Lazarus, editors. W. B. Saunders Co., Philadelphia. 252-268.

35. Editorial. 1991. Nitric oxide in the clinical arena. Lancet. 338:1560-1562.

36. Nava, E., R. M. J. Palmer, and S. Moncada. Inhibition of nitric oxide synthesis in septic shock: how much is beneficial? Lancet. 338:1555-1557. 